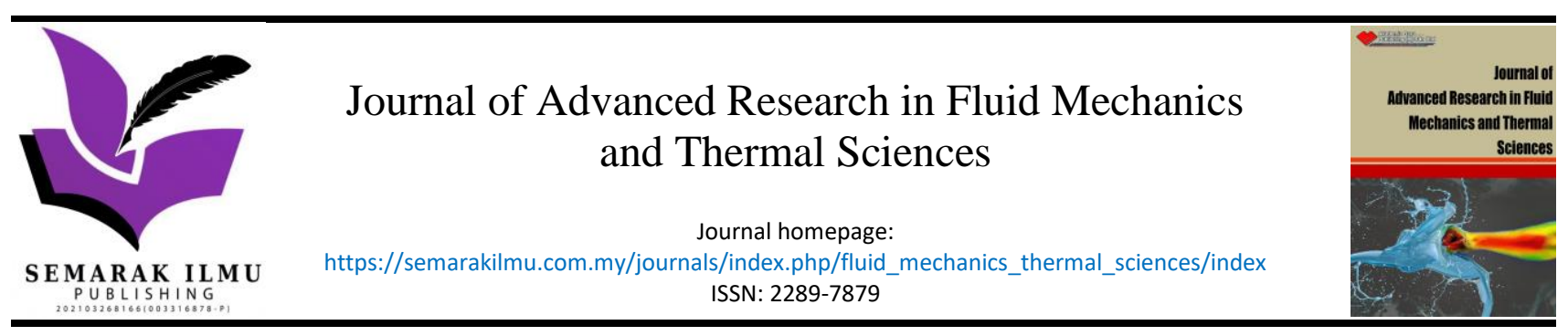

\title{
Homogeneous And Heterogeneous Reactions on The Peristalsis of Bingham Fluid with Variable Fluid Properties Through a Porous Channel
}

\author{
H. Balachandra' ${ }^{1}$, Choudhari Rajashekhar ${ }^{2}$, Hanumesh Vaidya ${ }^{3}$, Fateh Mebarek Oudina ${ }^{4}$, Gudekote \\ Manjunatha ${ }^{1,{ }^{*}}$, Kerehalli Vinayaka Prasad $^{3}$, Prathiksha ${ }^{1}$

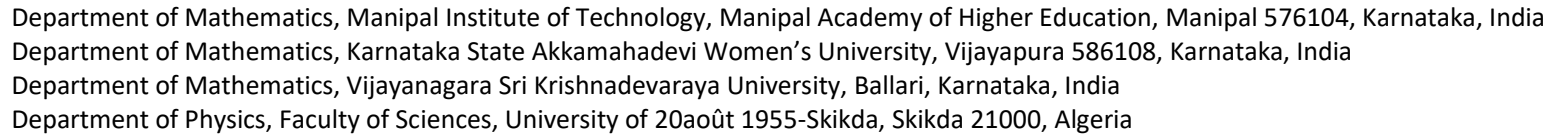

\section{ARTICLE INFO}

\section{Article history:}

Received 18 March 2021

Received in revised form 2 September 2021

Accepted 5 September 2021

Available online 6 November 2021

\section{Keywords:}

Homogeneous-Heterogeneous reactions; Grashof number; Variable liquid properties; Darcy number; Yield stress

\section{ABSTRACT}

The exploration addresses the effect of variable viscosity and thermal conductivity on the peristaltic mechanism of Bingham fluid. A two-dimensional non-uniform porous channel is considered for the fluid flow, which is assumed to be inclined. The impact of heat, slip conditions, wall properties, homogeneous and heterogeneous reactions are examined. The resulting nonlinear differential equations are solved by employing the perturbation method. The solutions acquired are analyzed and sketched through graphs that show that the variable viscosity renders a critical role in regulating the velocity of the fluid in the channel's central part. The stream function has been analyzed to observe the trapping phenomenon. Further, the obtained results find its application in understanding the flow of blood in micro arteries.

\section{Introduction}

The peristaltic mechanism is an inherent property caused by the contraction and expansion of the sinusoidal wave due to the flexible tube/channel walls. This mechanism is essential to understand bio-fluids' flow, such as blood, urine, eye drops, chyme movement, spermatozoa, etc. The mechanism was first investigated by Latham [1] using a Newtonian fluid model. He studied the spread of infection from bladder to kidney in the ureter. Shapiro et al., [2] investigated the peristaltic transport with a longer wavelength and lowered Reynolds number. In earlier days, research was carried out for Newtonian fluid. Later, Raju and Devanathan [3] studied the properties of peristaltic flow using nonNewtonian fluid. The mechanism of peristalsis of Herschel-Bulkley fluid was analyzed by Vajravelu et al., $[4,5]$ in an inclined tube, which investigates the flow characteristics of fluids. Jiménez-Lozano et al., [6] analyzed the peristalsis in a two-phase model to investigate the mechanism involved in ureteral biomechanics. Vaidya et al., [7] considered an inclined tube to study the transport of a

\footnotetext{
* Corresponding author.

E-mail address: manjunatha.g@manipal.edu
}

https://doi.org/10.37934/arfmts.88.3.119 
Bingham fluid and analyze the heat transfer effects during the peristalsis. Further, Manjunatha et al., [8] examined the function of convective boundary conditions, porous-walled tube, and variable liquid properties in the peristaltic movement of a non-Newtonian Bingham fluid.

The slip effects on biological fluids have gained many researchers' attention in modern times for their vast application in the field of medicine. The initial studies on the influence of slip effects on peristaltic transport are carried out by El-Shehawey and Husseny [9] and El-Shehawey et al., [10]. Awgichew and Radhakrishnamacharya [11] investigated the slip effect on the flow of a couple-stress fluid flowing in a symmetric two-dimensional channel with mild Stenosis. Akbar et al., [12] emphasized the impacts of slip effects on the sinusoidal flow of nanofluids. Later on, Ellahi and Hussain [13] considered a rectangular duct in their studies on a Jeffrey fluid's peristalsis. They obtained a closed-form solution of the problem and studied the effects of MHD and partial slip conditions on the fluid transport. Akbar [14] studied the impact of thermal slip and velocity on the MHD peristaltic motion of a Cu water nanofluid. Hayat et al., [15] analyzed the motion in a nonuniform permeable medium under the thermal slip conditions and radial magnetic field while accounting for the variable fluid viscosity. Recently, Manjunatha et al., [16] explored the effect of slip on the peristaltic flow of Rabinowitsch fluid inside an inclined non-slippery tube. Furthermore, the slip effects on peristaltic transport of different non-Newtonian fluids with different geometries are studied [17-22].

Over the past years, studies have shown that flow through porous media is also an essential factor in peristaltic studies, given its applications in various industrial and biological processes. Elshehawey et al., [23] obtained a precise form of the streamline function in their attempt to study the peristalsis in an asymmetric permeable conduit. Further, Alsaedi et al., [24] investigated the flow of an incompressible couple stress fluid in a permeable medium. They examined the effect of couple stress and permeability parameters on peristalsis inside a medium having pores. Nadeem et al., [25] studied the porous medium's influence on the peristalsis of a nanofluid. Ramesh and Devakar [26] studied the influence of heat and mass transfer in the peristalsis of a couple-stress fluid in a vertical asymmetric channel. They considered the effects of the magnetic field and homogeneous permeable medium. Recently, Rajashekhar et al., [27] examined the impact of variable liquid properties of the peristaltic transport through a porous medium.

In the past few years, the researchers noticed the importance of thermophysical properties in understanding various mechanisms associated with the human body. It is seen that in physiological fluids like blood and other liquids, these properties are not constant. In the variation in these properties, it is necessary to reflect on the variable thermal conductivity as well viscosity. The influence of varying viscosity was taken into consideration by Farooq et al., [28] in their peristaltic study on Jeffrey fluid. Abbasi et al., [29] revealed facts on the variable thermal conductivity and convective heat conditions in their investigations on the peristaltic motion of Carreau-Yasuda fluid. Both the properties of varying viscosity and thermal conductivity were reported by Baliga et al., [30] while investigating the peristalsis of Herschel-Bulkley fluid. In an attempt to investigate the effects of variable properties of a Bingham Fluid exhibiting porous peristaltic motion, Divya et al., [31] considered an inclined magnetic field. Due to its immense applicability in bioengineering and medicine, numerous researchers have studied its impact on various geometries [32-40].

The chemical reaction can be modified as either a homogeneous or heterogeneous process. It depends on whether they occur at an interface or as a single-phase volume reaction. The response is heterogeneous/homogeneous if it happens at an interface/in solution. There is the involvement of both homogeneous and heterogeneous reactions in several chemically reacting systems. Mention may be made to the processes occurring in catalysis, combustion, biochemical techniques, cooling towers, fog dispersion, crop-damaging through freezing, and many others. The study on modelling 
the peristaltic flow under the chemical reactions can be found in [41-43]. Recently, numerous studies have been carried on the investigation of non-Newtonian fluids in various geometries [44-47].

Having considered the various researchers and their work the author concludes that no attempts has been made to analyze the characteristic flow of a Bingham fluid during peristalsis through a nonuniform, inclined porous channel, where both variations of viscosity and thermal conductivity have also been considered. Moreover, the present study also investigates the homogeneous and heterogeneous reactions. The study also includes wall properties to understand the applications in industries and medicine better. Heat transfer is investigated with convective conditions. The mathematical model is solved by the method of perturbation. The consequences of relevant parameters on temperature, velocity, streamlines, and concentration are reported with graphs using MATLAB and the outcomes are explained.

\section{Formulation of the Problem}

A non-compressible non-Newtonian fluid flowing through an inclined non-uniform permeable channel is considered for analysis. The flow is governed by the Bingham Fluid model. The flow is induced by peristaltic waves moving with a constant speed $C$. The Cartesian coordinate system is chosen such that $x^{\prime}$ is the axial direction and $y^{\prime}$ is perpendicular to it.

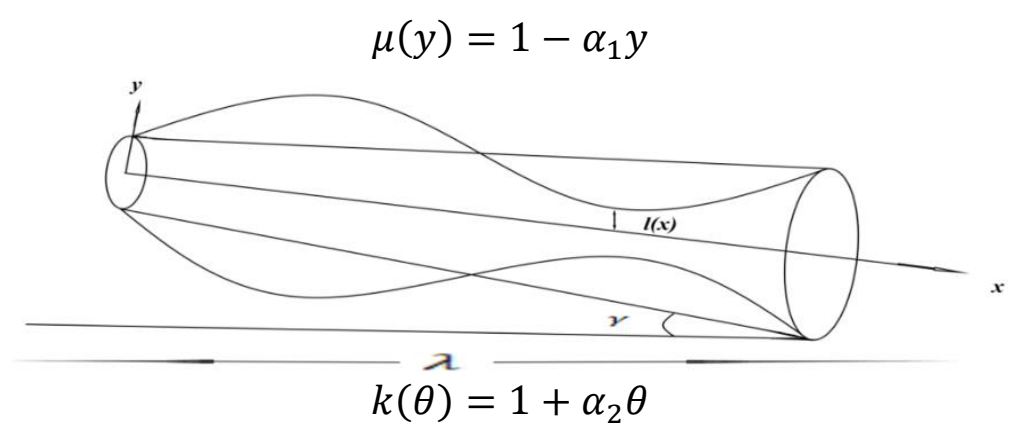

Fig. 1. Geometry of the physical model

We consider the homogeneous-heterogeneous reaction model between two chemical species $\hat{I}$ and $\hat{J}$ as defined below $[41,42]$

$\hat{I}+\hat{J} \rightarrow 3 \hat{J}$, Rate $=K_{c} I J^{2}$.

Further, single, isothermal and first order chemical reaction is considered on the catalyst surface. Thus, we have

$\hat{I} \rightarrow \hat{J}$, Rate $=K_{s} I$

where $I$ and $J$ are the concentration of $\hat{I}$ and $\hat{J}$ respectively, while $K_{c}$ and $K_{s}$ are the rate constants. Note that here both the reaction processes take place at the same temperature. 
The equations that govern the flow are

$$
\begin{aligned}
& \frac{\partial u^{\prime}}{\partial x^{\prime}}+\frac{\partial w^{\prime}}{\partial y^{\prime}}=0 \\
& \rho\left[\frac{\partial u^{\prime}}{\partial t^{\prime}}+u^{\prime} \frac{\partial u^{\prime}}{\partial x^{\prime}}+w^{\prime} \frac{\partial u^{\prime}}{\partial y^{\prime}}\right]=-\frac{\partial p^{\prime}}{\partial x^{\prime}}+\frac{\partial \tau^{\prime} x^{\prime} x^{\prime}}{\partial x^{\prime}}+\frac{\partial \tau^{\prime} x^{\prime} y^{\prime}}{\partial x^{\prime}}-\frac{\mu}{\kappa}\left(w^{\prime}+C\right)+\rho g \beta^{\prime} \sin \gamma\left(T_{1}-T_{0}\right), \\
& \rho\left[\frac{\partial w^{\prime}}{\partial t^{\prime}}+u^{\prime} \frac{\partial w^{\prime}}{\partial x^{\prime}}+w^{\prime} \frac{\partial w^{\prime}}{\partial y^{\prime}}\right]=-\frac{\partial p^{\prime}}{\partial x^{\prime}}+\frac{\partial \tau^{\prime} x^{\prime} y^{\prime}}{\partial x^{\prime}}+\frac{\partial \tau^{\prime} y^{\prime} y^{\prime}}{\partial x^{\prime}}-\frac{\mu}{\kappa}\left(u^{\prime}\right)+\rho g \beta^{\prime} \cos \gamma\left(T_{1}-T_{0}\right), \\
& \rho C_{p}\left[\frac{\partial T^{\prime}}{\partial t^{\prime}}+u^{\prime} \frac{\partial T^{\prime}}{\partial x^{\prime}}+w^{\prime} \frac{\partial T^{\prime}}{\partial y^{\prime}}\right]=k_{1}\left[\frac{\partial}{\partial x^{\prime}}\left(k\left(T^{\prime}\right) \frac{\partial T^{\prime}}{\partial x^{\prime}}\right)+\frac{\partial}{\partial y^{\prime}}\left(k\left(T^{\prime}\right) \frac{\partial T^{\prime}}{\partial y^{\prime}}\right)\right]+Q_{0}, \\
& \frac{d I}{d t}=M_{I}\left(\frac{\partial^{2} I}{\partial x^{\prime 2}}+\frac{\partial^{2} I}{\partial y^{\prime 2}}\right)-k c I J^{2}, \\
& \frac{d J}{d t}=M_{J}\left(\frac{\partial^{2} J}{\partial x^{\prime 2}}+\frac{\partial^{2} J}{\partial y^{\prime 2}}\right)+k c I J^{2},
\end{aligned}
$$

where $u^{\prime}, w^{\prime}$ are components of velocity in radial and axial directions respectively. $\rho$ is the fluid density, $P^{\prime}$ is the pressure, $\tau^{\prime} x^{\prime} x^{\prime}, \tau^{\prime} x^{\prime} y^{\prime}, \tau^{\prime} y^{\prime} y^{\prime}$ are extra stress components, while $k_{1}, T^{\prime}, C_{p}, M_{I}, M_{J}$, denotes thermal conductivity, temperature, specific heat at constant volume and mass diffusivity coefficient for homogeneous and heterogeneous reactions respectively.

The conditions at the boundary are

$$
\begin{aligned}
& \frac{\partial w^{\prime}}{\partial y^{\prime}}=\tau_{0} \text { at } y^{\prime}=0, \\
& w^{\prime}+\beta_{1} \frac{\partial w^{\prime}}{\partial y^{\prime}}=0 \text { at } y^{\prime}=H^{\prime}=l\left(x^{\prime}\right)+b^{\prime} \sin \left(\frac{2 \pi}{\lambda}\left(x^{\prime}-c t^{\prime}\right)\right) \\
& \frac{\partial T^{\prime}}{\partial y^{\prime}}=0 \text { at } y^{\prime}=0, \\
& -k_{1} \frac{\partial T^{\prime}}{\partial y^{\prime}}=\eta\left(T^{\prime}-T_{0}^{\prime}\right) \tau_{0} \text { at } y^{\prime}=H^{\prime}, \\
& I=I_{0} \text { at } y^{\prime}=0, \\
& M_{I} \frac{\partial I}{\partial y^{\prime}}=k_{s} I \text { at } y^{\prime}=H^{\prime},
\end{aligned}
$$




$$
\begin{aligned}
& J=J_{0} \text { at } y^{\prime}=0, \\
& M_{J} \frac{\partial J}{\partial y^{\prime}}=-k_{s} J \text { at } y^{\prime}=H^{\prime},
\end{aligned}
$$

where $H^{\prime}$ represents non-uniform wave wherein $l\left(x^{\prime}\right)$ is the non-uniform radius. The meanings of other variables used are given in the nomenclature section.

We now introducing the dimensionless quantities

$$
\begin{aligned}
& x=\frac{x^{\prime}}{\lambda}, y=\frac{y^{\prime}}{a^{\prime}}, w=\frac{w^{\prime}}{a^{\prime}}, u=\frac{\lambda u^{\prime}}{C a}, \tau_{x x}=\frac{a^{\prime} \tau^{\prime} x^{\prime} x^{\prime}}{C \mu}, \tau_{x y}=\frac{a^{\prime} \tau^{\prime} x^{\prime} y^{\prime}}{C \mu}, \tau_{y y}=\frac{a^{\prime} \tau^{\prime} y^{\prime} y^{\prime}}{C \mu}, t=\frac{c t^{\prime}}{\lambda}, \\
& \operatorname{Re}=\frac{a C \rho}{\mu}, p=\frac{a^{\prime 2} p^{\prime}}{C \lambda \mu}, \theta=\frac{T^{\prime}-T_{0}^{\prime}}{T_{1}-T_{0}}, \operatorname{Pr}=\frac{\mu C_{p}}{k_{1}}, D a=\frac{k_{1}}{a^{\prime}}, y_{p}^{\prime}=\frac{y_{p}}{a^{\prime}}, \beta=\frac{Q_{0} a^{\prime 2}}{k_{1}\left(T_{1}-T_{0}\right)} \\
& K_{s}=\frac{k_{s} I}{M_{I}}, \xi=\frac{M_{I}}{M_{J}}, G r=\frac{\rho \beta^{\prime} g a^{\prime 2}\left(T_{1}-T_{0}\right)}{\mu C}, g=\frac{I}{I_{0}}, f=\frac{I}{I_{0}}, \delta=\frac{a^{\prime}}{\lambda}, \varepsilon=\frac{b^{\prime}}{a^{\prime}}, \\
& E_{1}=\frac{-\sigma a^{\prime 3}}{\lambda \mu C}, E_{2}=\frac{m a^{\prime 3} C}{\lambda^{3} \mu}, E_{3}=\frac{a^{\prime 3} C}{\lambda^{3} \mu}, h=\frac{H^{\prime}}{a^{\prime}}=1+m x+\varepsilon \sin (2 \pi(x-t))
\end{aligned}
$$

Using Eq. (17) in Eq. (3)-(8) and by using low Reynolds number and long wavelength approximations, the non-dimensional governing equations take the form as below

$\frac{\partial p}{\partial x}=\frac{\partial \tau_{x y}}{\partial y}-\frac{1}{D a}(w+1)+G r \theta \sin y$,

$\frac{\partial p}{\partial y}=0$

$\frac{\partial}{\partial y}\left(k(\theta) \frac{\partial \theta}{\partial y}\right)+\beta=0$,

$\frac{1}{S c} \frac{\partial^{2}}{\partial y^{2}}=K f g^{2}$

$\frac{\xi}{S c} \frac{\partial^{2} g}{\partial y^{2}}=-K f g^{2}$

where $f, g$ are the dimensionless concentration of chemical species $\hat{I}$ and $\hat{J}$. Further, $\tau_{x y}$ denotes constitutive equation of Bingham fluid

$\tau_{x y}=\mu \dot{\gamma}+\tau_{0}, \quad \tau_{x y} \geq \tau_{0}$ 
$\dot{\gamma}=0, \quad \tau_{x y} \leq \tau_{0}$.

The dimensionless boundary conditions are

$$
\begin{aligned}
& \frac{\partial w}{\partial y}=\tau_{0} \quad \text { at } y=0, \\
& w+\beta_{1} \frac{\partial w}{\partial y}=0 \text { at } y=h, \\
& \frac{\partial \theta}{\partial y}=0 \quad \text { at } y=0, \\
& \theta+\beta_{2} \frac{\partial \theta}{\partial y}=0 \quad \text { at } \quad y=h, \\
& f=1 \quad \text { at } y=0, \\
& \frac{\partial f}{\partial y}=K_{s} f \quad \text { at } y=h, \\
& g=1 \quad \text { at } y=0, \\
& \frac{\partial g}{\partial y}=-K_{s} f \quad \text { at } \quad y=h,
\end{aligned}
$$

where $\beta_{1}$ and $\beta_{2}$ are velocity and temperature slip parameters respectively.

Assuming the diffusion constants $M_{I}$ and $M_{J}$ to be equal (i.e., $\xi=1$ ), which makes Eq. (29)-(32) take the following form

$$
f+g=1
$$

Using the above, Eq. (21) and Eq. (22) take the forms

$$
\frac{1}{S c} \frac{\partial^{2} f}{\partial y^{2}}=K f(1-f)^{2}
$$

with the boundary condition

$$
\begin{aligned}
& f=1 \quad \text { at } \quad y=0, \\
& \frac{\partial f}{\partial y}=K_{s} f \quad \text { at } \quad y=h,
\end{aligned}
$$


The varying viscosity across the wall channel thickness is

$$
\mu(y)=1-a_{1} y, \text { for } a_{1}<<1,
$$

where $a_{1}$ is the variable viscosity coefficient.

With respect to temperature, the thermal conductivity varies and is given by

$$
k(\theta)=1-a_{2} \theta, \text { for } a_{2}<<1,
$$

where $a_{2}$ is the coefficient of variable thermal conductivity.

\section{Solution to the Problem}

Eq. (18)-(22) are nonlinear equations. To solve these equations, we apply perturbation technique for smaller values of coefficients of variable viscosity and thermal conductivity respectively.

\subsection{Perturbation Solution}

The solution for velocity and temperature is obtained by solving Eq. (18) and Eq. (20) along with conditions at the boundary given by Eq. (25)-(28) using the perturbation series as follows

$$
\begin{aligned}
& w=\sum_{n=0}^{\infty}\left(a_{1}^{n} w_{n}\right) . \\
& \theta=\sum_{n=0}^{\infty}\left(a_{2}{ }^{n} \theta_{n}\right) .
\end{aligned}
$$

Also, the perturbation solution is obtained for the Eq. (34) for small parameter K.

$$
f=\sum_{n=0}^{\infty}\left(K^{n} f_{n}\right) .
$$

\subsubsection{Zeroth Order Solution}

$$
\begin{aligned}
& w_{0}=C_{1} \sin \frac{y}{\sqrt{D a}}+C_{2} \cos \frac{y}{\sqrt{D a}}-P D a+B_{1}\left(D a y^{4}-12 D a^{2} y^{2}+24 D a^{3}\right)+B_{2}\left(D a y^{2}-2 D a^{2}\right)+B_{3} D a . \\
& \theta_{0}=\left(\frac{\beta}{2} y^{2}+\beta \beta_{2} h+\frac{\beta}{2} h^{2}\right) . \\
& f_{0}=1+\frac{y K_{s}}{1-h K_{s}} .
\end{aligned}
$$




\subsubsection{First Order Solution}

$$
\begin{aligned}
& w_{1}=C_{3} \sin \frac{y}{\sqrt{D a}}+C_{4} \cos \frac{y}{\sqrt{D a}}+\left(G_{1}+G_{3}\right) y+G_{2} y^{3}+C_{1}\left(\left(y-\frac{1}{4}\right) \sin \frac{y}{\sqrt{D a}}+\left(\frac{y^{2}}{4 \sqrt{D a}}-\frac{\sqrt{D a}}{8}\right) \cos \frac{y}{\sqrt{D a}}\right) . \\
& +C_{2}\left(\frac{y}{4} \cos \frac{y}{\sqrt{D a}}-\left(\frac{y^{2}}{4 \sqrt{D a}}+\frac{\sqrt{D a}}{8}\right) \sin \frac{y}{\sqrt{D a}}\right) \\
& \theta_{0}=a_{2}\left(\frac{\beta}{2} y^{2}+\beta \beta_{2} h+\frac{\beta}{2} h^{2}\right) . \\
& f_{1}=\frac{S c K_{s}^{2}\left(y^{4}\left(-1+h K_{s}\right)\left(-5+5 k K_{s}-3 y K_{s}\right)-2 h^{3} y\left(10-5 h K_{s}+h^{2} K_{s}^{2}\right)\right)}{60\left(-1+h K_{s}^{4}\right)} .
\end{aligned}
$$

With the velocity expression, upper bound of the plug flow region is found with the help of following boundary condition

$$
\frac{\partial w}{\partial y}=0 \quad \text { at } \quad y=y_{p} .
$$

Using the above condition, we obtain the expression for stress in plug flow region. i.e.,

$$
\tau_{0}=\frac{\left(S_{1}+S_{2}\right)-a_{1}\left(S_{3}+S_{4}+S_{5}+S_{6}\right)}{S_{7}+\alpha_{1}\left(S_{8}+S_{9}+S_{10}+S_{11}\right)} .
$$

Using Eq. (42) and Eq. (45) we can also obtain the plug flow velocity when $y=y_{p}$ as

$$
w_{p}=X_{1} \cos \frac{y_{p}}{\sqrt{D a}}+X_{2} \sin \frac{y_{p}}{\sqrt{D a}}+X_{3}+X_{4}+a_{1} X_{5} .
$$

We now obtain the stream function using the following expression given below

$$
w=\frac{\partial \psi}{\partial y}, \quad u=-\frac{\partial \psi}{\partial x}
$$

with the boundary condition,

$$
\psi=0 \quad \text { at } \quad y=h .
$$

Then the expression for Stream function is given as

$$
\psi=\psi_{0}+\alpha_{1} \psi_{1}
$$


where,

$$
\begin{aligned}
& \psi_{0}=C_{2} \sqrt{D a} \sin \frac{y}{\sqrt{D a}}-C_{1} \sqrt{D a} \cos \frac{y}{\sqrt{D a}}-P D a y+B_{1}\left(D a \frac{y^{5}}{5}-12 D a^{2} \frac{y^{3}}{3}+24 D a^{3} y\right) \\
& +B_{2}\left(D a \frac{y^{3}}{3}-2 D a^{2} y\right)+B_{3} D a y \\
& \psi_{1}=C_{4} \sqrt{D a} \sin \frac{y}{\sqrt{D a}}-C_{3} \sqrt{D a} \cos \frac{y}{\sqrt{D a}}+C_{2}\left(-\frac{\sqrt{D a}}{4} y \sin \frac{y}{\sqrt{D a}}-\left(\frac{y^{2}}{4}+\frac{D a}{8}\right) \cos \frac{y}{\sqrt{D a}}\right) . \\
& +C_{1}\left(\frac{\sqrt{D a}}{2}\left(\frac{1}{4}+y\right) \cos \frac{y}{\sqrt{D a}}+\left(\frac{y^{2}}{4}+\frac{3 D a}{8}\right) \sin \frac{y}{\sqrt{D a}}\right)+\left(G_{1}+G_{2}\right) \frac{y^{2}}{2}+G_{2} \frac{y^{4}}{4}
\end{aligned}
$$

The expressions for $A_{1}, A_{2}, B_{1}, B_{2}, B_{3}, C_{i}(i=1,2,3,4), D_{j}(j=1,2, \ldots, 8), F_{k}(k=1,2, \ldots, 7), G_{1}, G_{2}, G_{3}$, $Q_{i}(I=1,2,3, \ldots, 11)$ and $X_{n}(n=1,2, \ldots, 5)$ are mentioned as follows

$$
\begin{aligned}
& P=\frac{\partial p}{\partial x}=8 \epsilon \pi^{3}\left(-\left(E_{1}+E_{2}\right) \cos (2 \pi(x-t))+E_{3} \frac{\sin (2 \pi(x-t))}{2 \pi}\right) \\
& A_{1}=-\frac{\beta}{2} \\
& A_{2}=\beta \beta_{2} h+\beta \frac{h^{2}}{2} \\
& B_{1}=\operatorname{Gr} A_{1}^{2} \alpha_{2} \sin \gamma \\
& B_{2}=\operatorname{Gr}\left(A_{1}+2 A_{1} A_{2} \alpha_{2}\right) \sin \gamma \\
& B_{3}=\operatorname{Gr} A_{2}^{2} \alpha_{2} \sin \gamma \\
& C_{1}=\tau_{0} \sqrt{D a} \\
& C_{2}=C_{1} F_{1}+F_{2}+F_{3}+F_{4} \\
& C_{3}=\sqrt{D a}\left(\tau_{0}-G_{1}-G_{3}-\frac{C_{2}}{8}+\frac{C_{1}}{4}\right) \\
& C_{4}=C_{3} F_{1}+F_{5}+C_{2} F_{6}+C_{1} F_{7} \\
& D_{1}=\frac{\beta_{1}}{\sqrt{D a}} \sin \frac{h}{\sqrt{D a}}-\cos \frac{h}{\sqrt{D a}} \\
& D_{2}=\frac{\beta_{1}}{\sqrt{D a}} \cos \frac{h}{\sqrt{D a}}+\sin \frac{h}{\sqrt{D a}} \\
& D_{3}=B_{2}\left(-D a h^{2}+2 D a^{2}-2 D a \beta_{1} h\right) \\
& D_{4}=B_{1}\left(D a h^{4}-12 D a^{2} h^{2}+24 D a^{3}+4 D a h^{3} \beta_{1}-24 D a \beta_{1} h\right) \\
& D_{5}=B_{3} D a-P D a \\
& D_{6}=\left(G_{1}+G_{3}\right)\left(h+\beta_{2}\right)+G_{2} h^{2}\left(h+3 \beta_{1}\right) \\
& D_{7}=\left(\frac{h}{4}+\frac{\beta_{1}}{4} \frac{h^{2}}{D a}+\frac{1}{8}\right) \cos \frac{h}{\sqrt{D a}}-\left(\frac{h^{2}}{4 \sqrt{D a}}+5 \frac{\sqrt{D a}}{8}-\frac{h}{4}\right) \sin \frac{h}{\sqrt{D a}} \\
& D_{8}=\left(\frac{h^{2}}{4 \sqrt{D a}}-\frac{\sqrt{D a}}{8}+\frac{3}{2} \beta_{1} h-\frac{\beta_{1}}{4 \sqrt{D a}}\right) \cos \frac{h}{\sqrt{D a}}+\left(h-\frac{1}{4}-\frac{\beta_{1}}{4} \frac{h^{2}}{D a}+7 \frac{\beta_{1}}{8}\right) \sin \frac{h}{\sqrt{D a}} \\
& F_{1}=\frac{D_{2}}{D_{1}}
\end{aligned}
$$




$$
\begin{aligned}
& F_{2}=-\frac{D_{3}}{D_{1}} \\
& F_{3}=\frac{D_{4}}{D_{1}} \\
& F_{4}=\frac{D_{5}}{D_{1}} \\
& F_{5}=\frac{D_{6}}{D_{1}} \\
& F_{6}=\frac{D_{7}}{D_{1}} \\
& F_{7}=\frac{D_{8}}{D_{1}} \\
& G_{1}=-144 B_{1} \sqrt{D a^{5}} \\
& G_{2}=16 B_{1} \sqrt{D a^{3}} \\
& G_{3}=4 B_{2} \sqrt{D a^{3}} \\
& S_{1}=B_{3} D a\left(24 D a y_{p}-y_{p}^{3}\right)-2 B_{2} D a \\
& S_{2}=-\frac{\left(F_{1}+F_{3}\right)}{\sqrt{D a}} \sin \frac{y_{p}}{\sqrt{D a}} \\
& S_{3}=\left(G_{1}+G_{3}\right)+G_{2} y_{p}^{2} \\
& S_{4}=\frac{\left(F_{2}+F_{3}\right)}{\sqrt{D a}}\left(\frac{y_{p}^{2}}{4 D a}+\frac{1}{8}\right) \cos \frac{y_{p}}{\sqrt{D a}}-\left(\frac{y_{p}}{4}+\frac{\sqrt{D a}}{2}\right) \sin \frac{y_{p}}{\sqrt{D a}} \\
& S_{5}=-\frac{\left(G_{1}+G_{3}\right)}{\sqrt{D a}} \cos \frac{y_{p}}{\sqrt{D a}} \\
& S_{6}=-\frac{D_{6}}{\sqrt{D a}} \sin \frac{y_{p}}{\sqrt{D a}} \\
& S_{7}=F_{1} \sin \frac{y_{p}}{\sqrt{D a}}+\cos \frac{y_{p}}{\sqrt{D a}} \\
& S_{8}=F_{1} \sqrt{D a}\left(\frac{y_{p}^{2}}{4 D a}+\frac{1}{8}\right) \cos \frac{y_{p}}{\sqrt{D a}}-\left(\frac{y_{p}}{4}+\frac{\sqrt{D a}}{2}\right) \sin \frac{y_{p}}{\sqrt{D a}} \\
& S_{9}=\sqrt{D a}\left(-\frac{y_{p}^{2}}{4 D a}+\frac{7}{8}\right) \sin \frac{y_{p}}{\sqrt{D a}}+\frac{3}{2} \sqrt{D a} y_{p}-\frac{1}{4} \cos \frac{y_{p}}{\sqrt{D a}} \\
& S_{10}=-\left(1+\frac{\sqrt{D a}}{4}-\frac{F_{1}}{8}\right) \cos \frac{y_{p}}{\sqrt{D a}} \\
& S_{11}=\left(\left(1+\frac{\sqrt{D a}}{4}-\frac{F_{1}}{8}\right) \frac{F_{1}}{\sqrt{D a}}+\frac{F_{1} F_{6}}{\sqrt{D a}}+\sqrt{D a} F_{7}\right) \sin \frac{y_{p}}{\sqrt{D a}} \\
& X_{1}=C_{2}+\alpha_{1}\left(C_{1}\left(\frac{y_{p}^{2}}{4 \sqrt{D a}}-\frac{\sqrt{D a}}{8}\right)+C_{2} \frac{y_{p}}{4}+C_{4}\right) \\
& X_{2}=C_{1}+\alpha_{1}\left(C_{1}\left(y-\frac{1}{4}\right)+C_{2} \frac{\sqrt{D a}}{8}+C_{3}\right) \\
& X_{3}=B_{2}\left(D a y_{p}^{2}-2 D a^{2}\right)+B_{3} D a-P D a \\
& X_{4}=B_{1}\left(D a y_{p}^{4}-12 D a^{2} y_{p}^{2}+24 D a^{3}\right) \\
& X_{5}=\left(G_{1}+G_{3}\right) y_{p}+G_{2} y_{p}^{3}-C_{2}
\end{aligned}
$$




\section{Results and Discussion}

In the current segment, the influence of important parameters on the temperature profiles, velocity profiles and stream function are discussed. The effects of rigidity parameter $\left(E_{1}\right)$, stiffness parameter $\left(E_{2}\right)$, viscous damping force parameter $\left(E_{3}\right)$, variable viscosity $\left(\alpha_{1}\right)$, Darcy number $(D a)$, velocity slip parameter $\left(\beta_{1}\right)$, temperature slip parameter $\left(\beta_{2}\right)$, non-uniformity parameter $(\mathrm{m})$, strength of heterogeneous reaction (Ks) and homogeneous reaction $(K)$, Schmidt number (Sc), are analyzed and discussed through graphs. MATLAB programming has been employed for the pictographic depictions of pertinent parameters of importance, taking the following values of parameters: $E_{1}=0.5, E_{2}=0.5, E_{3}=0.5, m=0.1, D a=0.2, \alpha_{1}=0.01, t=0.2, x=0.22, \beta_{1}=0.1, \beta_{2}=2, G r=1$, $y_{p}=0.3, \epsilon=0.3, K s=2, K=0: 2 ; S c=0: 5$ and $\gamma=\frac{\pi}{3}$.

\subsection{Velocity Profiles}

The influence of $\alpha_{1}, \beta, \beta_{1}, D a, \varepsilon, E_{1}, E_{2}, E_{3}, \gamma$ and $m$ on velocity are illustrated through Figure 2 . Each of these parameters are studied for their influence on velocity of the Bingham fluid model. The Figure 2(a) to Figure 2(j) clearly exhibit the parabolic profiles for velocity. Figure $2(\mathrm{a})$ is drawn to analyze the behavior of variable viscosity on the velocity profiles. The figure infers that, in the central part, growth of variable viscosity diminishes the velocity profiles of the fluid, while the behavior is contrast in the central part of the conduit for heat generating parameter Figure 2(b). The influence of velocity slip on velocity profile is represented in Figure 2(c) and we notice a decrease in velocity when the velocity slip parameter value increases. The velocity enhances for an increase in the porosity at the center of the channel (see Figure 2(d)). As the amplitude ratio rises, the central channel velocity enhances with larger estimation (Figure 2(e)). The effects of changes in the wall properties on the fluid are plotted. It is noticed that enhancing the values of $E_{1}$ and $E_{2}$ confronts an increase in the fluid velocity. With rise in the values of $E_{3}$, the velocity of the flow diminishes, and we can also notice that the change in velocity with respect to varying $E_{3}$ is so small that it can be negligible (see Figure 2(f) to Figure 2(h)). From the Figure 2(i) we observe that larger the inclination angle of the channel, faster the fluid moves. Also, as the channel becomes more non-uniform, velocity of the fluid reduces (see Figure 2(j)).
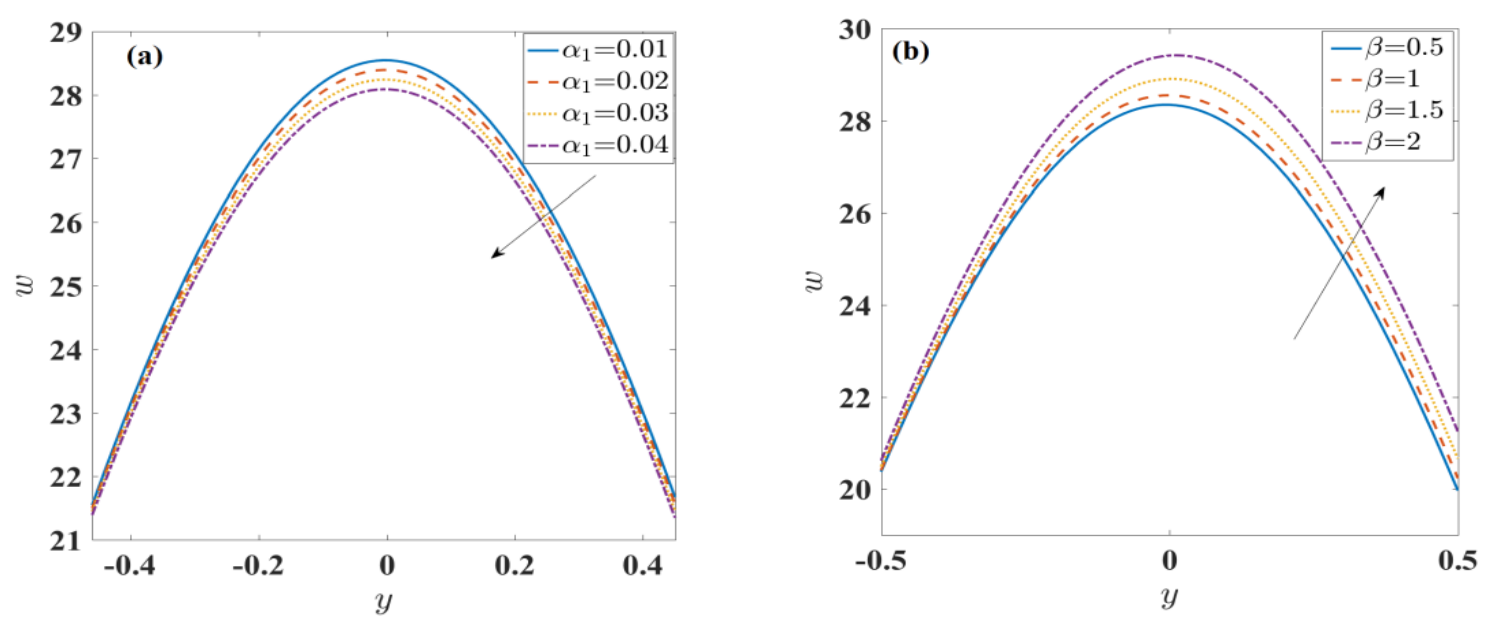

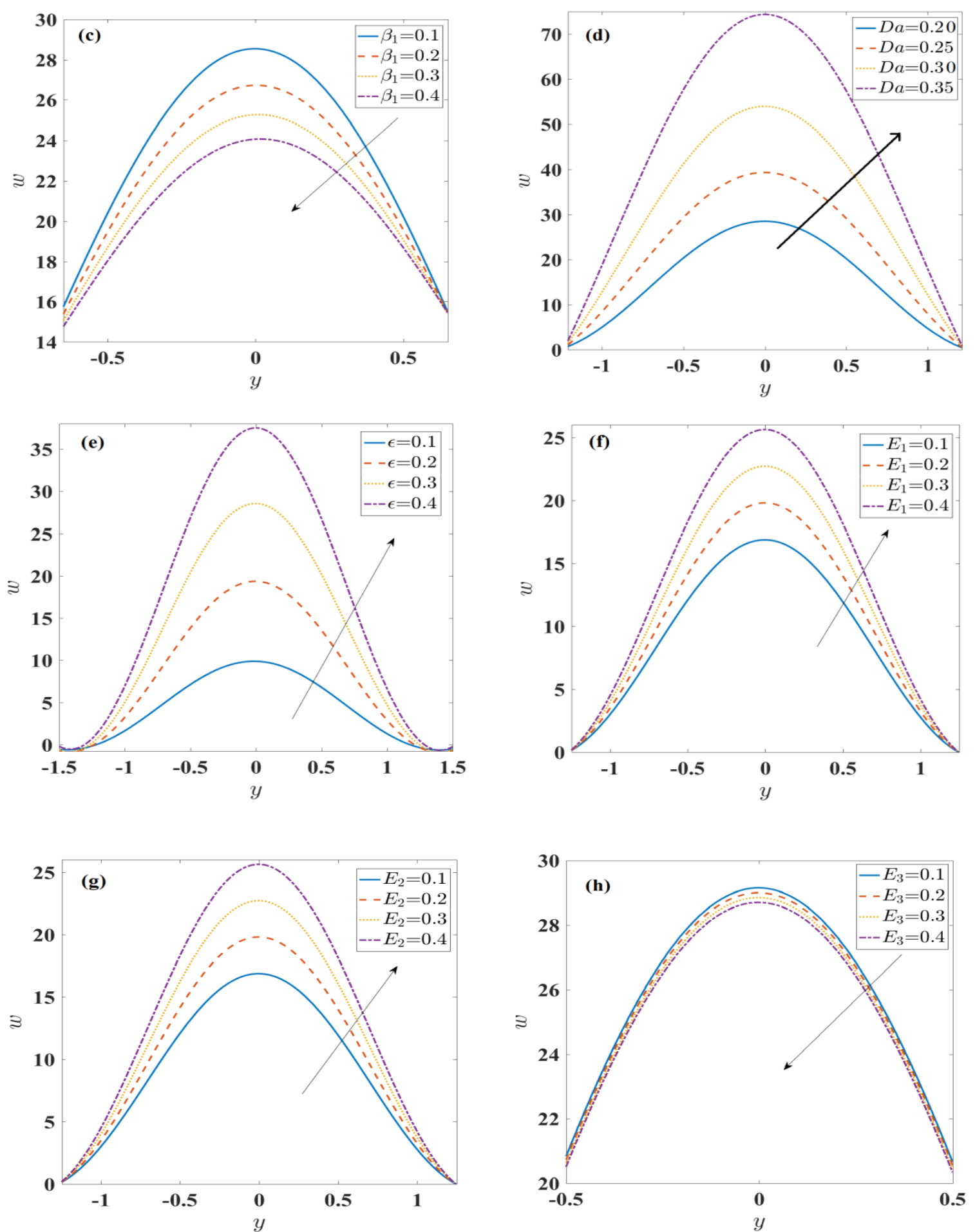

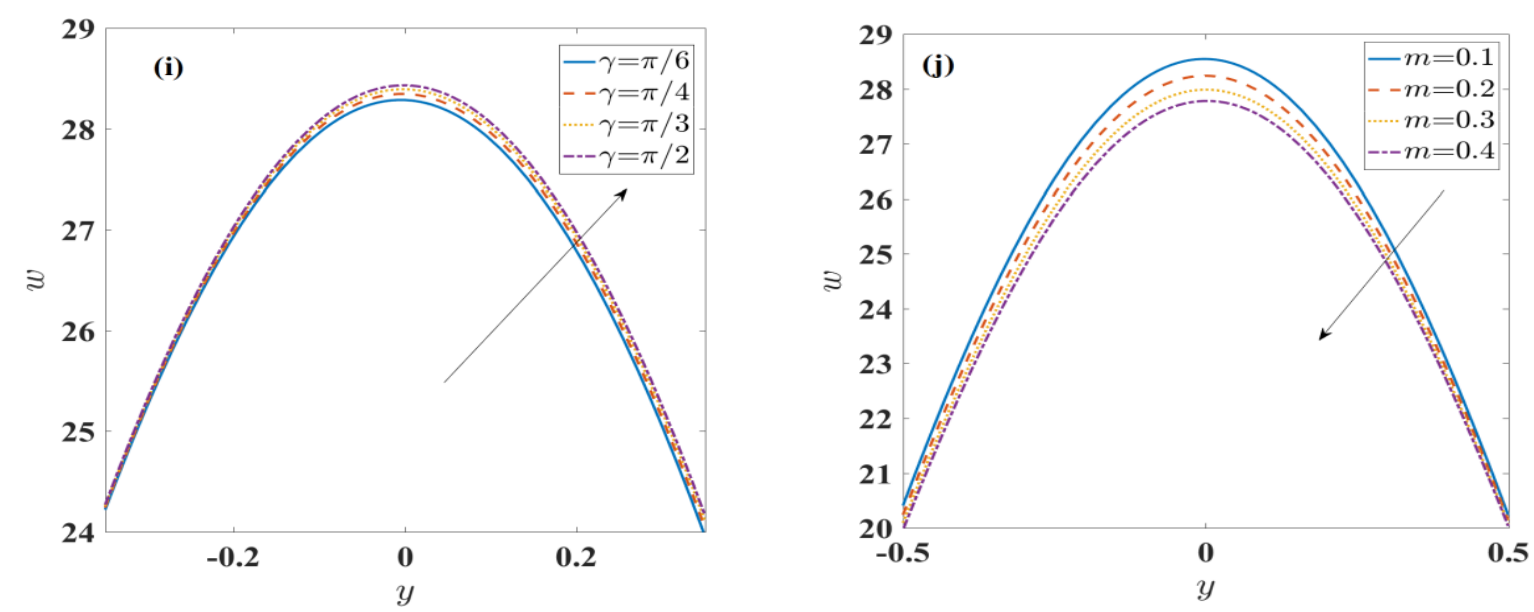

Fig. 2. Velocity profiles for varying (a) $\alpha_{1}$, (b) $\beta$, (c) $\beta_{1}$, (d) $\mathrm{Da}$, (e) $\varepsilon$ (f) $E_{1}$, (g) $E_{2}$, (h) $E_{3}$, (i) $\gamma$ and (j) $m$

\subsection{Temperature Profiles}

Graphical representations in Figure 3(a) to Figure 3(d) depict the variation in temperature profiles for varying physical parameters. The significance of $\beta$ in fluid temperature is shown in Figure 3(a), where it can be seen to increase the temperature with its rising value. In the flow of blood in arterioles, this behavior is reasonable due to the thickening of the boundary layer as heat is generated, which results in an appreciable rise in the temperature of the boundary layer. However, Figure $3(b)$ shows an enhancement in the fluid temperature with increasing variable thermal conductivity. The effect of thermal slip parameter can be seen to have an enhancement in temperature with an increase in these parameters (see Figure 3(c)). Figure 3(d) shows that the nonuniformity parameter enhances the temperature.

\subsection{Homogeneous and Heterogeneous Reaction Effects}

The effect of heterogeneous and homogeneous reactions $K_{S}$ and $K$, Schmidt number $S c$ and nonuniformity parameter $m$ on the concentration profiles are plotted in Figure 4(a) to Figure 4(d). From Figure 4(a) and Figure 4(b), it can be understood that while the parameter $K_{s}$ enhances the concentration with its increasing values, higher values of the parameter $K$ results in a diminution in the concentration. An increase in $S c$ would mean an increase in the molecular diffusion, thus resulting in a reduction in the concentration. This behavior is reflected in Figure 4(c). A contrary behavior of the non-uniformity parameter is depicted in Figure 4(d). 

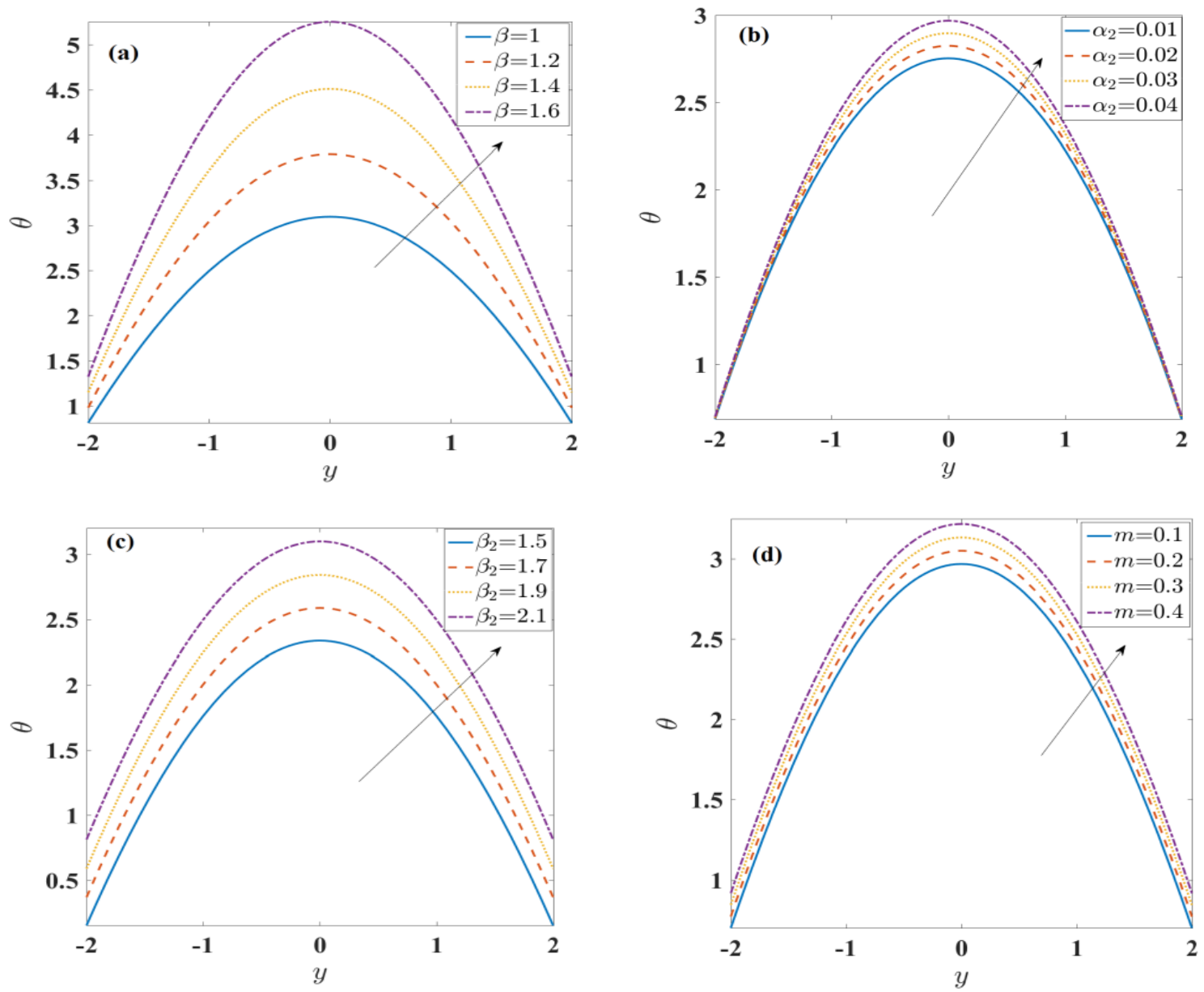

Fig. 3. Temperature profiles for varying (a) $\beta$, (b) $\alpha_{2}$, (c) $\beta_{2}$ and (d) $m$
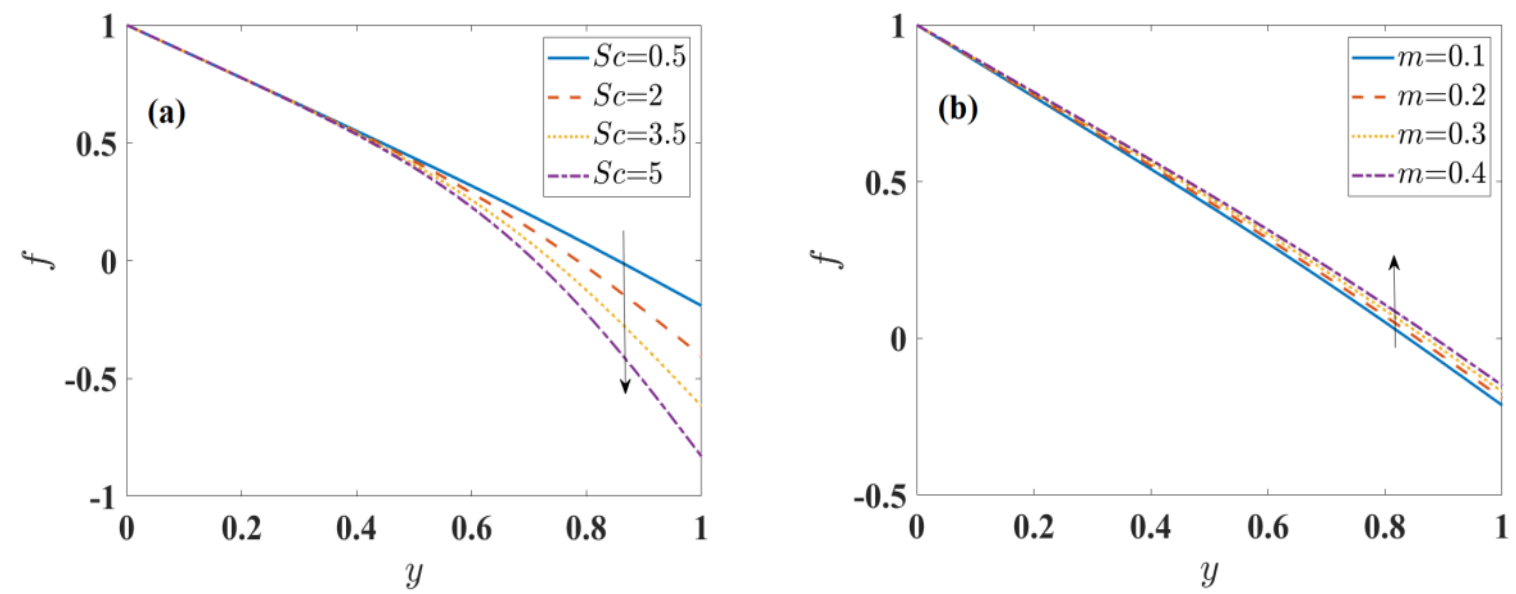

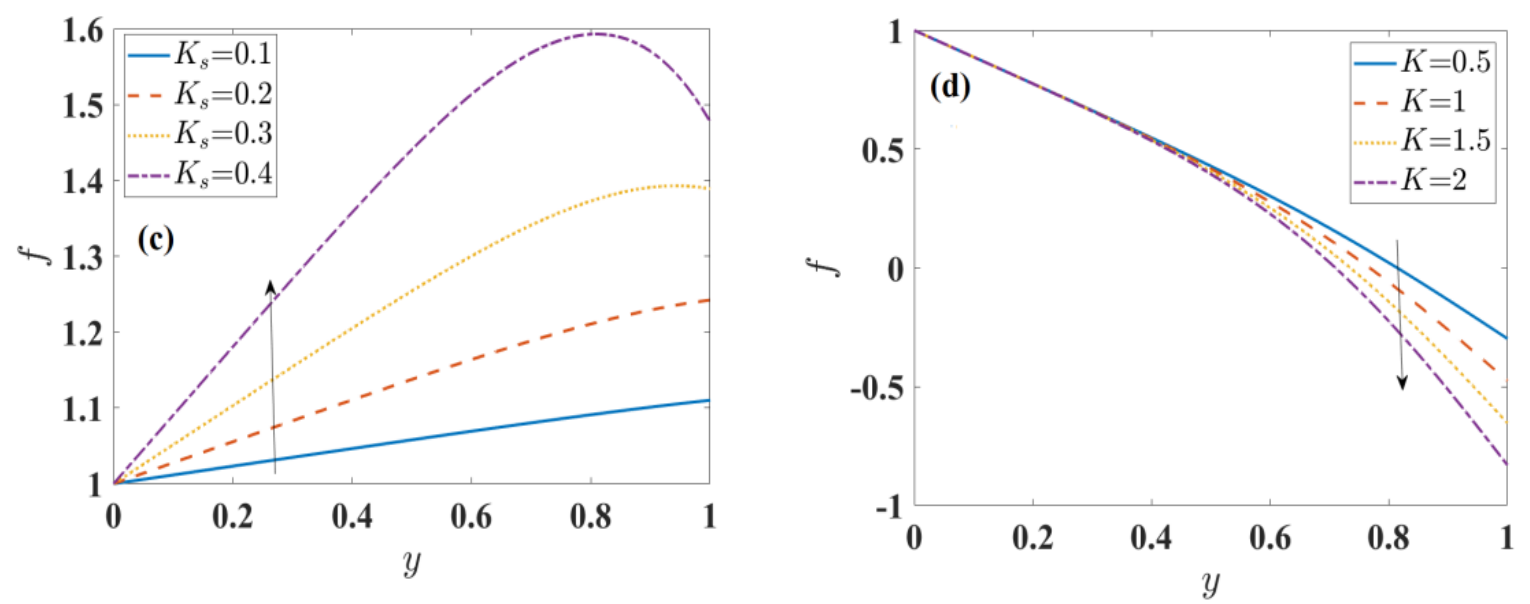

Fig. 4. Concentration profiles for varying (a) $S c$, (b) $m$, (c) $K_{S}$ and (d) $K$

\subsection{Trapping Phenomenon}

Trapping mechanism is an integral part of the peristaltic movement. This phenomenon occurs during peristalsis when few of its streamlines gets closed, resulting in the development of bolus that circulates on the inside and advances with speed of the peristaltic waves. This section attempts to study this interesting phenomenon of trapping through the plots of stream functions. Figure 5 is plotted to study the influence of variable viscosity on the pattern of stream function, in which it can be noticed that $\alpha_{1}$ contributes to a decrease in the bolus size. Effects of porous parameter $D a$ is seen to increase the bolus size during peristalsis (see Figure 6). While opposite behavior is seen for velocity slip parameter (Figure 7). A reduction in the bolus size is clearly seen as the value of Grashof number $(G r)$ increases in Figure 8.

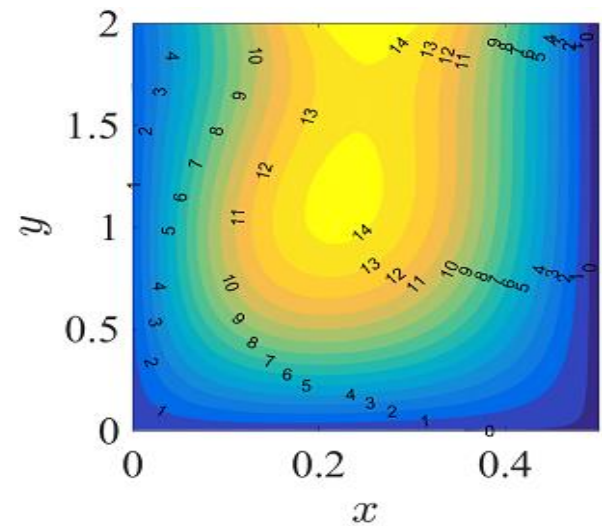

(a)

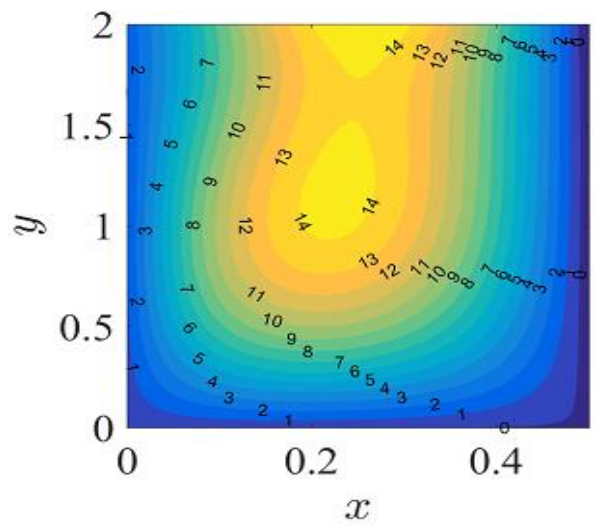

(b)

Fig. 5. Streamlines for varying (a) $\alpha_{1}=0.02$ and (b) $\alpha_{1}=0.03$ 


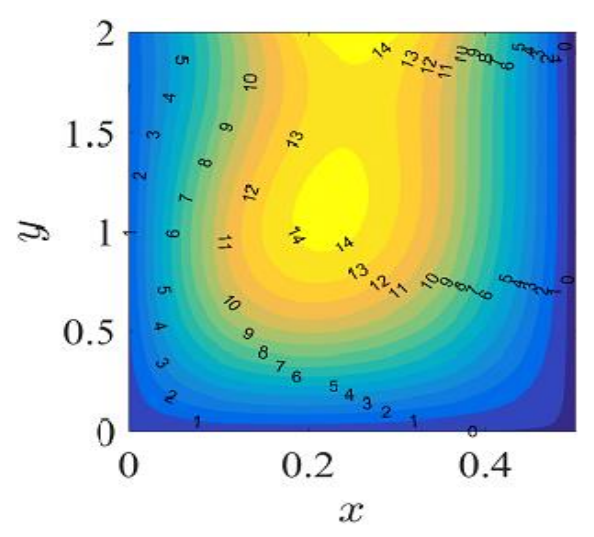

(a)

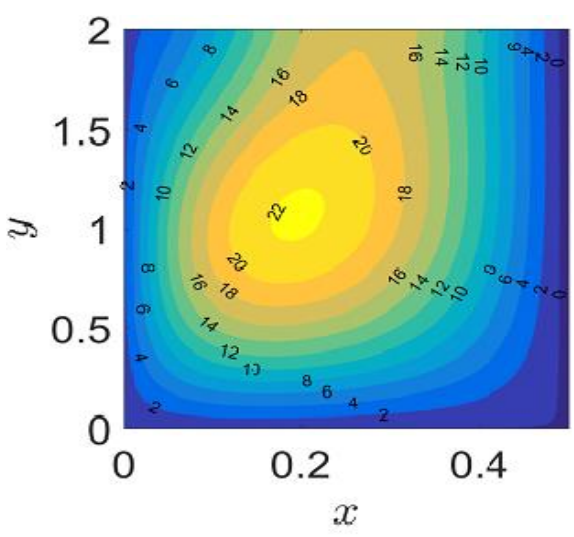

(b)

Fig. 6. Streamlines for varying (a) $D a=0.2$ and (b) $D a=0.25$

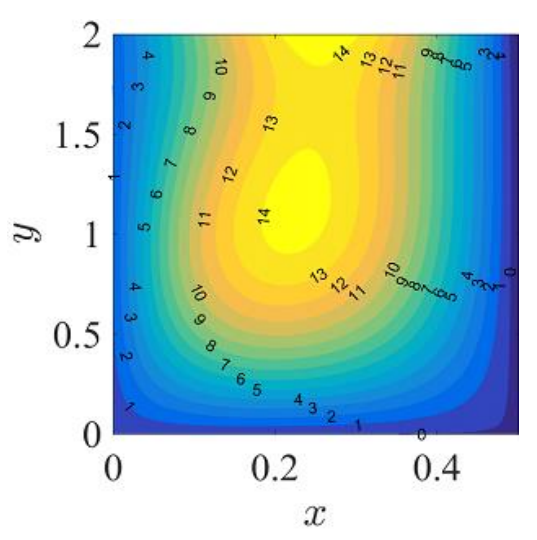

(a)

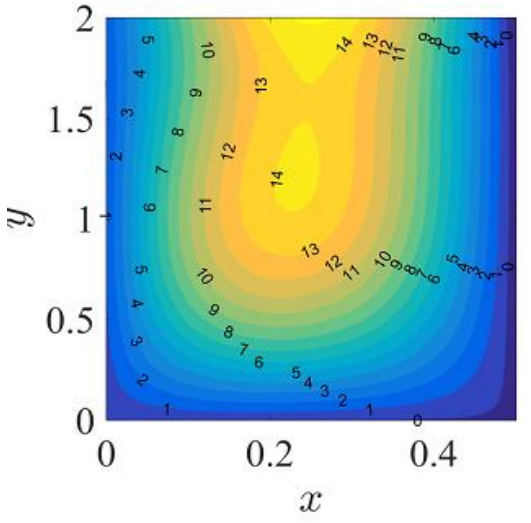

(b)

Fig. 7. Streamlines for varying (a) $\beta_{1}=0.1$ and (b) $\beta_{1}=0.15$

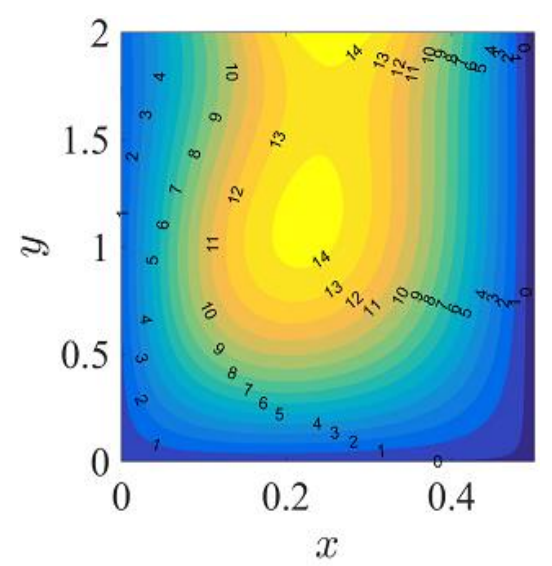

(a)

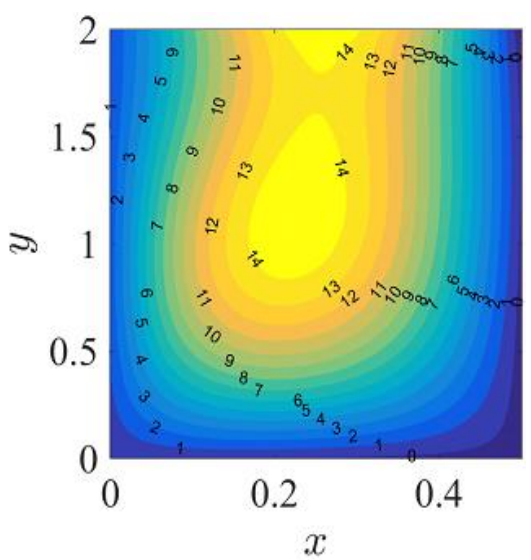

(b)

Fig. 8. Streamlines for varying (a) $G r=1$ and (b) $G r=3$

\section{Conclusion}

The current model investigates the consolidated impacts of convective boundary conditions and variable fluid properties on the peristaltic component of Bingham liquid in a slanted non-uniform channel. Some considerable findings from the current investigation are:

- Porous parameter aids for enhancing the velocity profiles while the slip parameter diminishes the velocity. 
- The variable thermal conductivity and heat generating parameters are increasing function of temperature.

- The wall parameters $E_{1}$ and $E_{2}$ increases the velocity of the fluid, whereas viscous damping force parameter $E_{3}$ reduces the velocity.

- The trapped bolus reduces its volume for higher values of velocity slip parameter and variable viscosity.

- The porous parameter is an aid for increasing the bolus formed during trapping.

\section{Acknowledgement}

The authors would like to thank the reviewers for the constructive and helpful comments that have led to a substantial improvement in the paper.

\section{References}

[1] Latham, Thomas Walker. "Fluid motions in a peristaltic pump." PhD diss., Massachusetts Institute of Technology, 1966.

[2] Shapiro, Ascher H., Michel Yves Jaffrin, and Steven Louis Weinberg. "Peristaltic pumping with long wavelengths at low Reynolds number." Journal of Fluid Mechanics 37, no. 4 (1969): $799-825$. https://doi.org/10.1017/S0022112069000899

[3] Raju, K. Kanaka, and Rathna Devanathan. "Peristaltic motion of a non-Newtonian fluid." Rheologica Acta 11, no. 2 (1972): 170-178. https://doi.org/10.1007/BF01993016

[4] Vajravelu, K., S. Sreenadh, and V. Ramesh Babu. "Peristaltic transport of a Herschel-Bulkley fluid in an inclined tube." International Journal of Non-Linear Mechanics 40, no. 1 (2005): 83-90. https://doi.org/10.1016/j.ijnonlinmec.2004.07.001

[5] Vajravelu, K., S. Sreenadh, and V. Ramesh Babu. "Peristaltic pumping of a Herschel-Bulkley fluid in a channel." Applied Mathematics and Computation 169, no. 1 (2005): 726-735. https://doi.org/10.1016/j.amc.2004.09.063

[6] Jiménez-Lozano, Joel, Mihir Sen, and Edmundo Corona. "Analysis of peristaltic two-phase flow with application to ureteral biomechanics." Acta Mechanica 219, no. 1 (2011): 91-109. https://doi.org/10.1007/s00707-010-0438-y

[7] Vaidya, Hanumesh, Choudhari Rajashekhar, Gudekote Manjunatha, and K. V. Prasad. "Effects of heat transfer on peristaltic transport of a Bingham fluid through an inclined tube with different wave forms." In Defect and Diffusion Forum, vol. 392, pp. 158-177. Trans Tech Publications Ltd, 2019. https://doi.org/10.4028/www.scientific.net/DDF.392.158

[8] Manjunatha, Gudekote, C. Rajashekhar, Hanumesh Vaidya, and K. V. Prasad. "Peristaltic mechanism of Bingham liquid in a convectively heated porous tube in the presence of variable liquid properties." Special Topics \& Reviews in Porous Media: An International Journal 10, no. 2 (2019): $187-201$. https://doi.org/10.1615/SpecialTopicsRevPorousMedia.2019026973

[9] El Shehawey, E. F., and S. Z. A. Husseny. "Effects of porous boundaries on peristaltic transport through a porous medium." Acta Mechanica 143, no. 3 (2000): 165-177. https://doi.org/10.1007/BF01170946

[10] El-Shehawy, E. F., N. T. El-Dabe, and I. M. El-Desoky. "Slip effects on the peristaltic flow of a non-Newtonian Maxwellian fluid." Acta Mechanica 186, no. 1 (2006): 141-159. https://doi.org/10.1007/s00707-006-0343-6

[11] Awgichew, Gurju, and G. Radhakrishnamacharya. "Effects of Slip Condition and Peripheral Layer on Couple Stress Fluid Flow through a Channel with Mild Stenosis." International Journal of Mechanical and Mechatronics Engineering 7, no. 2 (2013): 299-304.

[12] Akbar, Noreen Sher, S. Nadeem, and Zafar Hayat Khan. "Thermal and velocity slip effects on the MHD peristaltic flow with carbon nanotubes in an asymmetric channel: application of radiation therapy." Applied Nanoscience 4 , no. 7 (2014): 849-857. https://doi.org/10.1007/s13204-013-0265-2

[13] Ellahi, R., and F. Hussain. "Simultaneous effects of MHD and partial slip on peristaltic flow of Jeffery fluid in a rectangular duct." Journal of Magnetism and Magnetic Materials 393 (2015): $284-292$. https://doi.org/10.1016/j.jmmm.2015.05.071

[14] Akbar, Noreen Sher. "Influence of thermal and velocity slip on the peristaltic flow of $\mathrm{Cu}$-water nanofluid with magnetic field." Applied Nanoscience 6, no. 3 (2016): 417-423. https://doi.org/10.1007/s13204-015-0444-4

[15] Hayat, T., S. Farooq, A. Alsaedi, and B. Ahmad. "Influence of variable viscosity and radial magnetic field on peristalsis of copper-water nanomaterial in a non-uniform porous medium." International Journal of Heat and Mass Transfer 103 (2016): 1133-1143. https://doi.org/10.1016/i.ijheatmasstransfer.2016.07.101 
[16] Manjunatha, Gudekote, Choudhari Rajashekhar, Hanumesh Vaidya, K. V. Prasad, and Oluwole Daniel Makinde. "Effects wall properties on peristaltic transport of rabinowitsch fluid through an inclined non-uniform slippery tube." In Defect and Diffusion Forum, vol. 392, pp. 138-157. Trans Tech Publications Ltd, 2019. https://doi.org/10.4028/www.scientific.net/DDF.392.138

[17] Choudhari, Rajashekhar, Manjunatha Gudekote, and Naveen Choudhari. "Analytical Solutions on the Flow of blood with the Effects of Hematocrit, Slip and TPMA in a porous tube." Journal of Advanced Research in Fluid Mechanics and Thermal Sciences 47, no. 1 (2018): 201-208.

[18] Gudekote, Manjunatha, and Rajashekhar Choudhari. "Slip effects on peristaltic transport of Casson fluid in an inclined elastic tube with porous walls." Journal of Advanced Research in Fluid Mechanics and Thermal Sciences 43, no. 1 (2018): 67-80.

[19] Prasad, Kerehalli Vinayaka, Hanumesh Vaidya, Gudekote Manjunatha, Kuppalapalle Vajravelu, Choudhari Rajashekhar, and V. Ramanjini. "Influence of Variable Transport Properties on Casson Nanofluid Flow over a Slender Riga Plate: Keller Box Scheme." Journal of Advanced Research in Fluid Mechanics and Thermal Sciences 64, no. 1 (2019): 19-42.

[20] Gudekote, Manjunatha, Hanumesh Vaidya, Divya Baliga, Rajashekhar Choudhari, Kerehalli Vinayaka Prasad, and Viharika Viharika. "The effects of convective and porous conditions on peristaltic transport of non-Newtonian fluid through a non-uniform channel with wall properties." Journal of Advanced Research in Fluid Mechanics and Thermal Sciences 63, no. 1 (2019): 52-71.

[21] Choudhari, Rajashekhar, Manjunatha Gudekote, Hanumesh Vaidya, and Kerehalli Vinayaka Prasad. "Peristaltic flow of Herschel-Bulkley fluid in an elastic tube with slip at porous walls." Journal of Advanced Research in Fluid Mechanics and Thermal Sciences 52, no. 1 (2018): 63-75.

[22] Bilal, S., M. Sohail, R. Naz, M. Y. Malik, and M. Alghamdi. "Upshot of ohmically dissipated Darcy-Forchheimer slip flow of magnetohydrodynamic Sutterby fluid over radiating linearly stretched surface in view of Cash and Carp method." Applied Mathematics and Mechanics 40, no. 6 (2019): 861-876. https://doi.org/10.1007/s10483-0192486-9

[23] Elshehawey, E. F., Nabil T. Eldabe, E. M. Elghazy, and Abdelhalim Ebaid. "Peristaltic transport in an asymmetric channel through a porous medium." Applied Mathematics and Computation 182, no. 1 (2006): $140-150$. https://doi.org/10.1016/i.amc.2006.03.040

[24] Alsaedi, A., Nasir Ali, Dharmendra Tripathi, and Tasawar Hayat. "Peristaltic flow of couple stress fluid through uniform porous medium." Applied Mathematics and Mechanics 35, no. 4 (2014): $469-480$. https://doi.org/10.1007/s10483-014-1805-8

[25] Nadeem, S., Arshad Riaz, R. Ellahi, and Noreen Sher Akbar. "Mathematical model for the peristaltic flow of nanofluid through eccentric tubes comprising porous medium." Applied Nanoscience 4, no. 6 (2014): $733-743$. https://doi.org/10.1007/s13204-013-0249-2

[26] Ramesh, K., and M. Devakar. "Effects of heat and mass transfer on the peristaltic transport of MHD couple stress fluid through porous medium in a vertical asymmetric channel." Journal of Fluids 2015 (2015). https://doi.org/10.1155/2015/163832

[27] Rajashekhar, C., G. Manjunatha, Hanumesh Vaidya, B. Divya, and K. Prasad. "Peristaltic flow of Casson liquid in an inclined porous tube with convective boundary conditions and variable liquid properties." Frontiers in Heat and Mass Transfer (FHMT) 11 (2018). https://doi.org/10.5098/hmt.11.35

[28] Farooq, S., M. Awais, Moniza Naseem, T. Hayat, and B. Ahmad. "Magnetohydrodynamic peristalsis of variable viscosity Jeffrey liquid with heat and mass transfer." Nuclear Engineering and Technology 49, no. 7 (2017): 13961404. https://doi.org/10.1016/i.net.2017.07.013

[29] Abbasi, F. M., T. Hayat, and B. Ahmad. "Numerical analysis for peristaltic transport of Carreau-Yasuda fluid with variable thermal conductivity and convective conditions." Journal of Central South University 22, no. 11 (2015): 4467-4475. https://doi.org/10.1007/s11771-015-2994-8

[30] Baliga, Divya, Manjunatha Gudekote, Rajashekhar Choudhari, Hanumesh Vaidya, and Kerehalli Vinayaka Prasad. "Influence of velocity and thermal slip on the peristaltic transport of a herschel-bulkley fluid through an inclined porous tube." Journal of Advanced Research in Fluid Mechanics and Thermal Sciences 56, no. 2 (2019): 195-210.

[31] Divya, B. B., G. Manjunatha, C. Rajashekhar, Hanumesh Vaidya, and Kerehalli Vinayaka Prasad. "Effects of inclined magnetic field and porous medium on peristaltic flow of a Bingham fluid with heat transfer." Journal of Applied and Computational Mechanics (2019).

[32] Manjunatha, G., C. Rajashekhar, Hanumesh Vaidya, K. V. Prasad, O. D. Makinde, and J. U. Viharika. "Impact of variable transport properties and slip effects on MHD Jeffrey fluid flow through channel." Arabian Journal for Science and Engineering 45, no. 1 (2020): 417-428. https://doi.org/10.1007/s13369-019-04266-y 
[33] Hussain, Q., S. Asghar, T. Hayat, and A. Alsaedi. "Peristaltic transport of hydromagnetic Jeffrey fluid with temperature-dependent viscosity and thermal conductivity." International Journal of Biomathematics 9, no. 02 (2016): 1650029. https://doi.org/10.1142/S1793524516500297

[34] Sohail, Muhammad, Rahila Naz, Zahir Shah, Poom Kumam, and Phatiphat Thounthong. "Exploration of temperature dependent thermophysical characteristics of yield exhibiting non-Newtonian fluid flow under gyrotactic microorganisms." AIP Advances 9, no. 12 (2019): 125016. https://doi.org/10.1063/1.5118929

[35] Vaidya, Hanumesh, C. Rajashekhar, G. Manjunatha, and K. V. Prasad. "Rheological properties and peristalsis of Rabinowitsch fluid through compliant porous walls in an inclined channel." Journal of Nanofluids 8, no. 5 (2019): 970-979. https://doi.org/10.1166/jon.2019.1664

[36] Vaidya, Hanumesh, Rajashekhar Choudhari, Manjunatha Gudekote, and Kerehalli Vinayaka Prasad. "Effect of variable liquid properties on peristaltic transport of Rabinowitsch liquid in convectively heated complaint porous channel." Journal of Central South University 26, no. 5 (2019): 1116-1132. https://doi.org/10.1007/s11771-019$\underline{4075-x}$

[37] Sohail, Muhammad, Umar Nazir, Yu-Ming Chu, Hussam Alrabaiah, Wael Al-Kouz, and Phatiphat Thounthong. "Computational exploration for radiative flow of Sutterby nanofluid with variable temperature-dependent thermal conductivity and diffusion coefficient." Open Physics 18, no. 1 (2020): 1073-1083. https://doi.org/10.1515/phys$\underline{2020-0216}$

[38] Manjunatha, G., C. Rajashekhar, Hanumesh Vaidya, K. V. Prasad, O. D. Makinde, and J. U. Viharika. "Impact of variable transport properties and slip effects on MHD Jeffrey fluid flow through channel." Arabian Journal for Science and Engineering 45, no. 1 (2020): 417-428. https://doi.org/10.1007/s13369-019-04266-y

[39] Abdelsalam, Sara I., and M. Sohail. "Numerical approach of variable thermophysical features of dissipated viscous nanofluid comprising gyrotactic micro-organisms." Pramana 94, no. 1 (2020): 1-12. https://doi.org/10.1007/s12043-020-1933-x

[40] Sohail, Muhammad, Zahir Shah, Asifa Tassaddiq, Poom Kumam, and Prosun Roy. "Entropy generation in MHD Casson fluid flow with variable heat conductance and thermal conductivity over non-linear bi-directional stretching surface." Scientific Reports 10, no. 1 (2020): 1-16. https://doi.org/10.1038/s41598-020-69411-2

[41] Chaudhary, M. A., and J. H. Merkin. "A simple isothermal model for homogeneous-heterogeneous reactions in boundary-layer flow. I Equal diffusivities." Fluid Dynamics Research 16, no. 6 (1995): 311. https://doi.org/10.1016/0169-5983(95)00015-6

[42] Kameswaran, P. K., S. Shaw, P. V. S. N. Sibanda, and P. V. S. N. Murthy. "Homogeneous-heterogeneous reactions in a nanofluid flow due to a porous stretching sheet." International Journal of Heat and Mass Transfer 57, no. 2 (2013): 465-472. https://doi.org/10.1016/i.ijheatmasstransfer.2012.10.047

[43] Bilal, S., Muhammad Sohail, and Rahila Naz. "Heat transport in the convective Casson fluid flow with homogeneousheterogeneous reactions in Darcy-Forchheimer medium." Materials and Structures 15, no. 6 (2019): 1170-1189. https://doi.org/10.1108/MMMS-11-2018-0202

[44] Sohail, Muhammad, Rahila Naz, and Sara I. Abdelsalam. "Application of non-Fourier double diffusions theories to the boundary-layer flow of a yield stress exhibiting fluid model." Physica A: Statistical Mechanics and its Applications 537 (2020): 122753. https://doi.org/10.1016/j.physa.2019.122753

[45] Sohail, Muhammad, and Rahila Naz. "Modified heat and mass transmission models in the magnetohydrodynamic flow of Sutterby nanofluid in stretching cylinder." Physica A: Statistical Mechanics and its Applications 549 (2020): 124088. https://doi.org/10.1016/j.physa.2019.124088

[46] Naz, Rahila, Sana Tariq, Muhammad Sohail, and Zahir Shah. "Investigation of entropy generation in stratified MHD Carreau nanofluid with gyrotactic microorganisms under Von Neumann similarity transformations." The European Physical Journal Plus 135, no. 2 (2020): 1-22. https://doi.org/10.1140/epjp/s13360-019-00069-0

[47] Sohail, M., R. Naz, and Sara I. Abdelsalam. "On the onset of entropy generation for a nanofluid with thermal radiation and gyrotactic microorganisms through 3D flows." Physica Scripta 95, no. 4 (2020): 045206. https://doi.org/10.1088/1402-4896/ab3c3f 